\title{
Depletion of the proteasome subunit PSMA7 inhibits colorectal cancer cell tumorigenicity and migration
}

\author{
XIAO-TONG HU ${ }^{1,2}$, WEI CHEN ${ }^{3}$, FU-BIAO ZHANG ${ }^{1,2}$, QING-LAN SHI ${ }^{1,2}$, \\ JIAN-BIN HU ${ }^{4}$, SHU-MIN GENG ${ }^{3}$ and CHAO HE $\mathrm{HE}^{1,2,3}$ \\ ${ }^{1}$ Biomedical Research Center, Sir Run Run Shaw Hospital, Zhejiang University; \\ ${ }^{2}$ Key Laboratory of Biotherapy of Zhejiang Province; Departments of ${ }^{3}$ Colorectal Surgery and \\ ${ }^{4}$ Radiotherapy, Sir Run Run Shaw Hospital, Zhejiang University, Hangzhou, Zhejiang, P.R. China
}

Received June 2, 2009; Accepted July 20, 2009

DOI: 10.3892/or_00000561

\begin{abstract}
Colorectal cancer is one of the most common causes of cancer-related deaths throughout the world. Recently, we reported that proteasome subunit PSMA7 located on 20q13 amplicon was overexpressed and associated with liver metastasis of colorectal cancer. The results indicate that PSMA7 may play an important role in the colorectal cancer progression and provide a unique target site for the development of therapeutic drugs. However, it is unknown how aberrant PSMA7 activation critically regulates the metastatic behavior of colorectal cancer cells. To investigate the role of PSMA7 in the progression of colorectal cancer, we employed the RNA interference technology to knock down the PSMA7 gene in human colon cancer cell line RKO and analyzed its effect and explored the involved mechanisms. Depletion of PSMA7 by shRNA in RKO cells inhibited their anchorageindependent growth and cell invasion and migration. Moreover, PSMA7 depletion was able to strongly suppress the in vivo tumorigenic ability of RKO cells. These effects may be induced by inhibiting CD44 expression directly or indirectly. Genetic or pharmacological inhibition of PSMA7 may therefore be a beneficial strategy in the treatment of colorectal cancer patients.
\end{abstract}

\section{Introduction}

Colorectal cancer (CRC) is one of the most common causes of cancer-related deaths throughout the world. Although advances in treatment have reduced patient morbidity, the survival rates of CRC have remained low. Molecular investigations have provided evidence that multiple genetic alterations are involved in colorectal tumorigenesis. A molecular genetic model of

Correspondence to: Dr Chao He, Biomedical Research Center, Sir Run Run Shaw Hospital, Zhejiang University, 3 Qingchun East Road, Hangzhou, Zhejiang 310016, P.R. China

E-mail: drhe_srrsh@sina.com

Key words: PSMA7, RNA interference, RKO, colon cancer preferential sequence has been proposed (1). However, even with the present knowledge of the cellular and molecular mechanisms of CRC, no biological parameter can predict the behavior of cancers. Recently, we reported that the proteasome subunit PSMA7 located on 20q13 amplicon was overexpressed and associated with liver metastasis of colorectal cancer (2). The results indicate that PSMA7 may play an important role in the colorectal cancer progression and provide a unique target site for the development of therapeutic drugs. However, it is unknown how PSMA7 critically regulates the metastatic behavior of colorectal cancer cells.

To investigate the role of PSMA7 in the progression of colorectal cancer, we employed the RNA interference technology to knock down the PSMA7 gene in human colon cancer cell line RKO and analyzed its effect and explored the involved mechanisms. We found that depletion of PSMA7 by shRNA in RKO cells inhibited their anchorage-independent growth and cell migration and invasion. Moreover, PSMA7 depletion was able to strongly suppress the in vivo tumorigenic ability of RKO cells. These effects may be induced by inhibiting CD44 expression directly or indirectly.

\section{Materials and methods}

Cell cultures. The human colorectal cancer cell line RKO was cultured in Dulbecco's modified Eagle's medium (DMEM) (Gibco BRL, Grand Island, NY) containing 10\% fetal bovine serum (FBS) (Biochrom, Berlin, Germany), $100 \mathrm{U} / \mathrm{ml}$ penicillin (Sigma-Aldrich, St. Louis, MO, USA) and $100 \mu \mathrm{g} / \mathrm{ml}$ streptomycin (Sigma-Aldrich) under humidified conditions in $95 \%$ air and $5 \% \mathrm{CO}_{2}$ at $37^{\circ} \mathrm{C}$.

Construction of PSMA7 shRNA. The target sequences against human PSMA7 cDNA (NM_002792) were designed at the Ambion website (www.ambion.com) and aligned by BLAST to avoid off-target match. The pSilencer ${ }^{\mathrm{TM}}$ siRNA expression vectors kit (Ambion, Austin, TX, USA) was used according to the manufacturer's protocol to produce shRNA expression cassettes (SECs). The most effective SEC targeting PSMA7 mRNA (5'-CTG CAG GAT GAA AGA ACA G-3') was cloned into the pSilencer ${ }^{\mathrm{TM}}$ 2.1-U6 hygro vector. Cells transfected with the pSilencer hygro plasmid expressing PSMA7 target-specific shRNA was compared to cells 
transfected with the corresponding pSilencer hygro-negative control plasmid supplied with the kit.

Cell transfection and stable colony selection. For transfection, RKO cells were plated in 24-well plates at a density of $1 \times 10^{5}$ cells per well and allowed to grow overnight to $90-95 \%$ confluency. The next day, the cells were transfected with the mixture of $0.8 \mu \mathrm{g}$ DNA (PSMA7 shRNA plasmid or negative control plasmid) and $2 \mu \mathrm{l}$ Lipofectamine 2000 (Invitrogen, USA) in $100 \mu 1$ serum-free medium according to the manufacturer's instructions. To produce stably transfected cells, after transfection with the shRNA expression construct, $1500 \mu \mathrm{g} / \mathrm{ml}$ of hygromycin (Roche, Gemany) was added at $48 \mathrm{~h}$ to the medium (DMEM $+15 \%$ FBS $)$. The cells were left in selective medium for two weeks after which they were trypsinized and recultured in selective medium for propagation.

Western blot analysis. Cell extracts were prepared with lysis buffer. Total protein concentration was measured by using a BCA protein assay reagent kit (Pierce, Rockford, IL, USA). Ten micrograms of sample per well were loaded on a $12 \%$ SDS-PAGE and transferred electrophoretically to a nitrocellulose membrane. After having been blocked in TBS-T buffer with $5 \%$ dry milk for $1 \mathrm{~h}$ at room temperature, the membranes were incubated sequentially with primary antibody overnight at $4^{\circ} \mathrm{C}$ and HRP-conjugated secondary antibody (1:1000, Santa Cruz, CA, USA) for $2 \mathrm{~h}$ at room temperature in TBS-T milk. Primary antibodies were used as follows: anti-PSMA7 mouse monoclonal antibody (clone 1A10-3G12, Abnova, Taipei, Taiwan); anti-CD44 rabbit polyclonal antibody (1:1000, Cell Signaling, Beverly, MA, USA); antiHIF-1 $\alpha$ mouse monoclonal antibody (1:1000, BD Bioscience, San Jose, CA, USA) and anti-Actin mouse monoclonal antibody (1:1000, Santa Cruz Biotechnologies, USA). Detection was carried out using the ECL kit (Amersham Biosciences, Piscataway, NJ, USA) and the blots were developed using a Fujifilm Las-4000 Imaging System.

MTT assay. RKO cells were subjected to the MTT [3-(4,5dimethylthiazol-2-yl)-2,5-diphenyltetrazolium bromide] assay, according to the manufacturer's protocol (SigmaAldrich).

Cell cycle analysis. Cell cycle analysis was performed with Propidium Iodide (PI) staining. Cells were collected in $5 \mathrm{ml}$ PBS and spun for $5 \mathrm{~min}$ at $200 \mathrm{x} \mathrm{g}$. Cells were resuspended in $0.5 \mathrm{ml}$ PBS and further fixed in ice-cold $70 \%$ ethanol for at least $2 \mathrm{~h}$. Fixed cells were next centrifuged at $200 \mathrm{x}$ g for $5 \mathrm{~min}$ and the pellet was resuspended in $1 \mathrm{ml}$ of freshly prepared PI staining solution $(20 \mu \mathrm{g} / \mathrm{ml} \mathrm{PI} / 0.1 \%$ Triton/0.2 mg DNase-free RNase A). Samples were kept for $30 \mathrm{~min}$ at room temperature and maintained at $4{ }^{\circ} \mathrm{C}$ overnight in the dark.

Soft agar assay. For the soft agar colony formation assay, $\sim 500$ cells were suspended in medium containing $0.3 \%$ low melt agarose, seeded into a 6-well plate that was overlaid with $0.5 \%$ low melt agarose and allowed to grow for two weeks at $37^{\circ} \mathrm{C}$ in $5 \% \mathrm{CO}_{2}$. The colonies containing $>50$ cells were counted under a microscope. Three wells were analyzed for each experiment.
Wound-healing assay. Cell migration was studied by a scratch wound-healing assay. Cells $\left(5 \times 10^{5}\right.$ per well) were seeded in 6-well plates and allowed to adhere for $24 \mathrm{~h}$. The cells were treated with $10 \mu \mathrm{g} / \mathrm{ml}$ Mitomycin C (Sigma) for $3 \mathrm{~h}$, washed with PBS and then simply wounded with a pipette tip. Fresh, full medium was added and the cells were allowed to close the wound for $48 \mathrm{~h}$. Photographs were taken every $24 \mathrm{~h}$ at the same position of the wound. The experiment was done in triplicate.

Cell invasion and migration assay. RKO cells transfected with either PSMA7 shRNA or scrambled shRNA were trypsinized and resuspended in DMEM containing $1 \%$ FBS at a density of $1 \times 10^{6}$ cells $/ \mathrm{ml}$. In all, $100 \mu 1$ of the cell suspension was plated on 24-well Transwell (Costar, Corning, NY, USA) coated with $100 \mu \mathrm{l}$ of Matrigel $(250 \mu \mathrm{g} / \mathrm{ml})$. DMEM $(600 \mu \mathrm{l})$ containing $10 \%$ FBS was placed in the lower chamber. After incubation for $24 \mathrm{~h}$, cells remaining in upper chamber were removed carefully by cotton swab and the membrane was cut off by an operating knife. The side facing lower chamber was stained with $0.05 \%$ crystal violet and attached cells were counted under a light microscope. The experiment was performed three times.

Cell migration assays were performed in a similar manner but without the Matrigel coating.

In vivo subcutaneous tumor model. All of the in vivo experimental protocols were approved by the Animal Care Committee of Sir Run Run Shaw Hospital, Zhejiang University. Viable RKO cells $\left(2 \times 10^{6}\right.$ cells $\left./ 0.2 \mathrm{ml} / \mathrm{mouse}\right)$ were injected subcutaneously into right flank of 5-week-old female BALB/c nude mice ( 6 mice per group). Tumor volume was assessed every 3 days for 5 weeks. Tumor volume was calculated by the following formula: (short diameter) ${ }^{2} \mathrm{x}$ (long diameter) $/ 2$.

Statistical analysis. Statistical analysis was performed in SPSS 11.0 for Windows (SPSS Inc., Chicago, IL, USA). Data are presented as means \pm SD and were compared by Student's t-test. $\mathrm{P}<0.05$ was considered to be of statistical significance.

\section{Results}

PSMA7 shRNA transfection efficiently silences PSMA7 protein expression in RKO colon cancer cells. To evaluate inhibition of PSMA7 protein expression, Western blot analysis was performed. A significant reduction in PSMA7 protein was detected in PSMA7 shRNA-transfected cells as compared with parental and scrambled shRNA-transfected cells. In stable PSMA7 shRNA-transfected clone 1, PSMA7 was significantly reduced, while in stable PSMA7 shRNA-transfected clone 2, PSMA7 was efficiently silenced. While no difference was observed among the parental and the scrambled shRNAtransfected cells (Fig. 1). The stable PSMA7 shRNAtransfected clone 2 was selected for further investigation.

PSMA7 knockdown suppressed RKO cell motility but not the proliferation and the cell cycle transition. The MTT and cell cycle analysis results suggested that PSMA7 knockdown did not affect the proliferation and the cycle transition in RKO cells (data not shown). To explore the roles of PSMA7 in the 


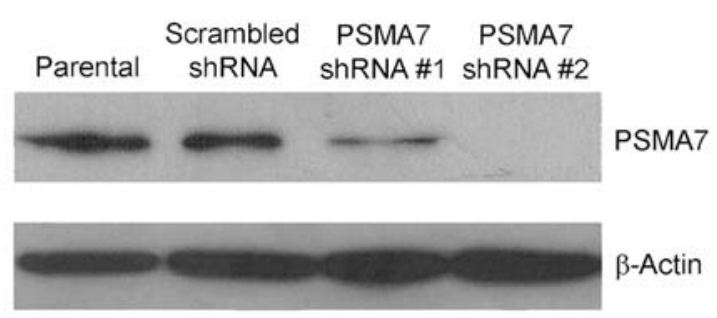

Figure 1. PSMA7 shRNA transfection efficiently silences the PSMA7 gene in RKO colon cancer cells. Western blot analysis showed PSMA7 protein expression level of parental, PSMA7 shRNA-transfected and scrambled shRNA-transfected RKO cells. PSMA7 protein expression in PSMA7 shRNA-transfected cells was reduced (clone \#1) or totally inhibited (clone \#2) as compared with parental and scrambled shRNA-transfected cells. regulation of cell motility, wound-healing assay was performed. The cells were pretreated with Mitomycin C, which inhibits cell division, so that the difference in motility was not affected by differences in cell proliferation rates. The PSMA7 shRNAtransfected cells took $>48$ h to close a scratch wound, whereas the scrambled shRNA-transfected cells took $<48 \mathrm{~h}$ to heal a similar size wound (Fig. 2A).

In addition, PSMA7 depletion significantly inhibited RKO cell invasion and migration (Fig. 2B and D). The number of invading or migrated cells of the PSMA7-transfected cells was significantly reduced as compared with the control (Fig. 2C and $\mathrm{E})$.

Tumor-suppressive function of PSMA7 depletion in RKO cells in vitro and in vivo. To study whether the transforming
(A)
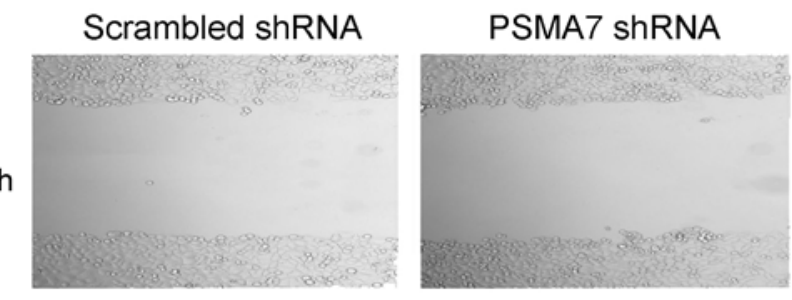

$24 \mathrm{~h}$
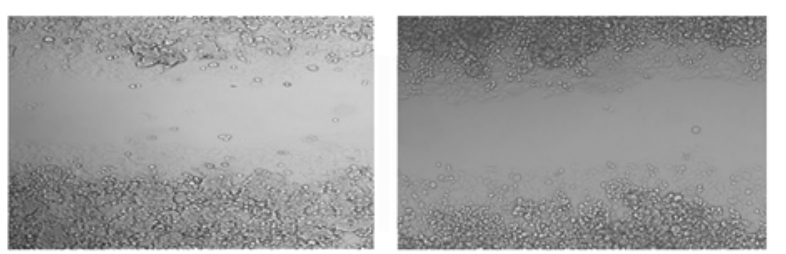

$48 \mathrm{~h}$
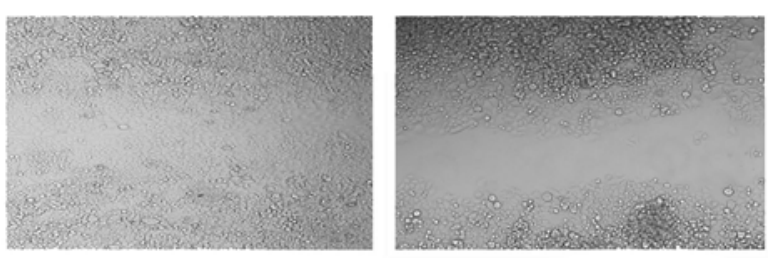

(B)

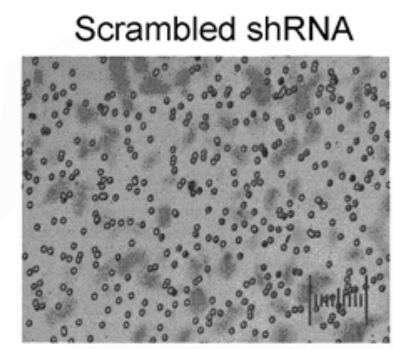

(D)
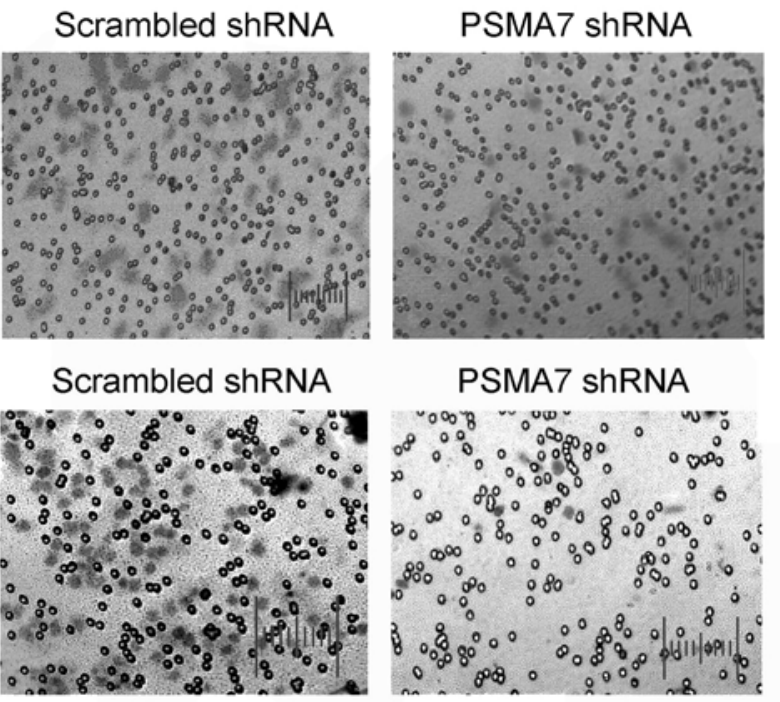

PSMA7 ShRNA

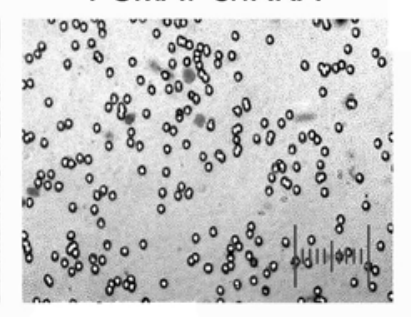

(C)

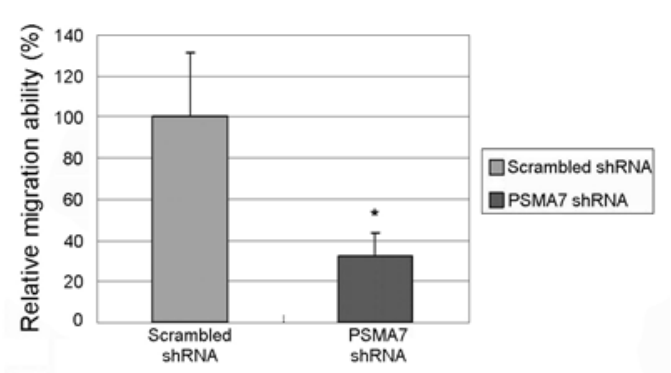

(E)

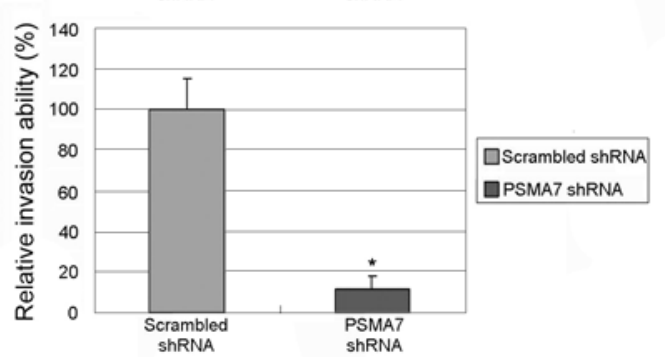

Figure 2. PSMA7 knockdown suppresses the motility of RKO cells. (A) Wound healing assay for stable PSMA7 shRNA-transfected and scrambled shRNAtransfected RKO cells. The pictures were taken every $24 \mathrm{~h}$ (original magnification: x100). (B and D) Migration and invasion of the PSMA7 shRNA-transfected and scrambled shRNA-transfected RKO cells was assayed by 24-Transwell system. The pictures of migrated cells were taken $24 \mathrm{~h}$ after seeding (original magnification: x100). (C and E) Quantitative analysis of cell migration and invasion. The number of cells that had migrated or invased was counted in five representative high power fields (HPFs) per Transwell insert. Errors bars represented the standard error of the mean. *Indicated P<0.05 when compared to the control cells. Scale bar corresponds to $100 \mu \mathrm{m}$ in all panels. 
(A)

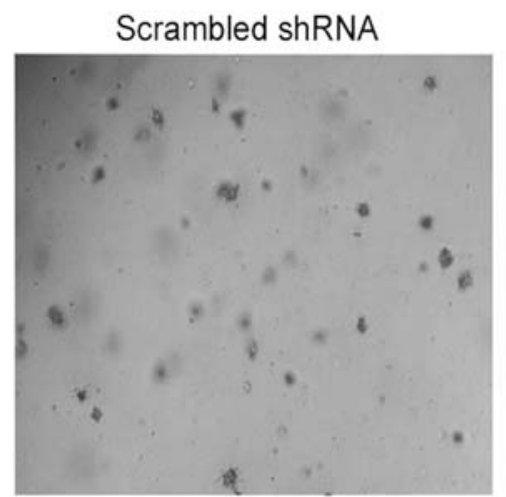

(B)

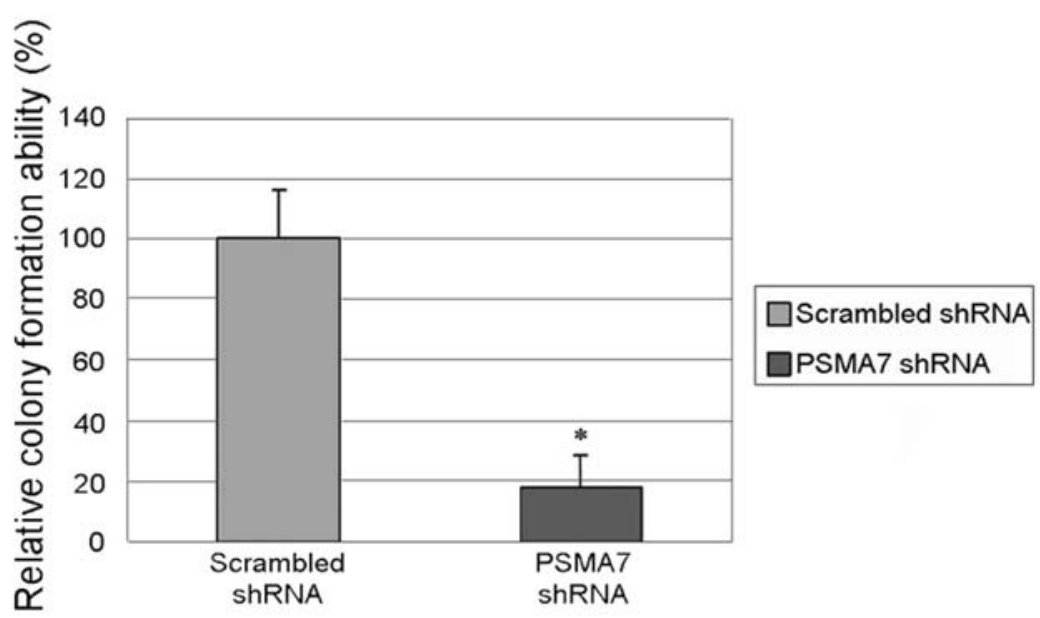

(C)

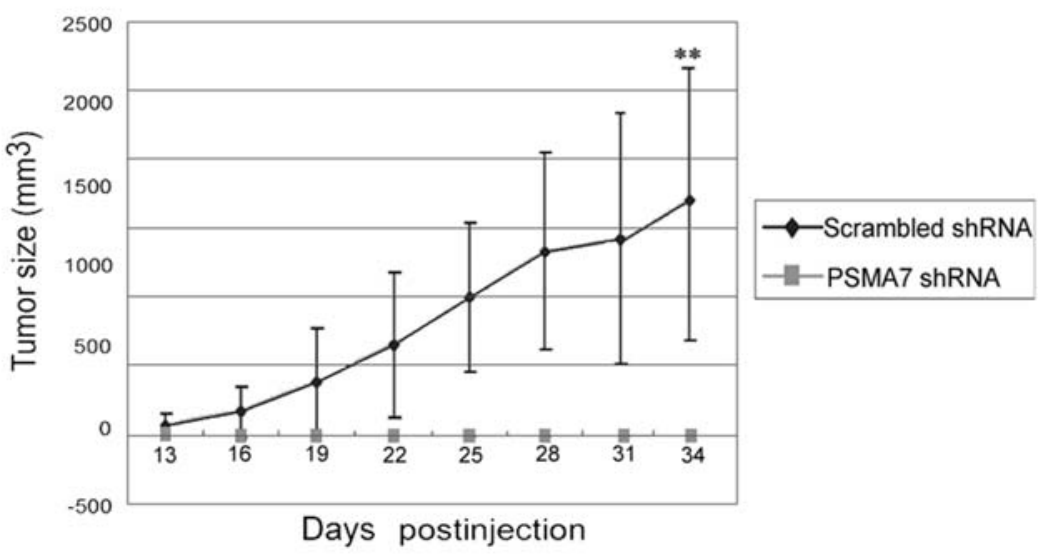

Figure 3. Tumor-suppressive function of PSMA7 depletion in RKO cells in vitro and in vivo. (A) The stable PSMA7 shRNA-transfected and scrambled shRNAtransfected RKO cells were assayed for colony formation $\left(3 \times 10^{4}\right.$ cells per $60 \mathrm{~mm}$ dish) in soft agar. Colonies were counted and photographed after 4 weeks of growth (original magnification: x400). These results are representative of three independent experiments. A separate cluster with $>50$ cells was regarded as a clone. (B) Quantitative analysis of colony formation efficiency. Error bars represent the standard error of the mean. "Indicates $\mathrm{P}<0.05$ when compared to the control cells. (C) Tumor growth curves of stable PSMA7 shRNA-transfected and scrambled shRNA-transfected RKO cells in nude mice by tumor xenograft experiment. ${ }^{* *}$ Indicate $\mathrm{P}<0.001$ when compared to the control cells.

ability of RKO cells could be reversed by PSMA7 depletion, anchorage-independent soft agar assay was performed. The results are shown in Fig. 3A. The scrambled shRNAtransfected cells grew and formed large colonies within 4 weeks. In contrast, PSMA7 shRNA-transfected cells failed to grow in soft agar plate and most of them remained as single cells. PSMA7 knockdown significantly inhibited the anchorage-independent growth of RKO cells $(\mathrm{P}<0.05)$ (Fig. 3B).
To further explore the in vivo tumor-suppressive ability of PSMA7 depletion, tumor formation in nude mouse was tested by injection of stable PSMA7 shRNA-transfected cells $(n=6)$ and scrambled shRNA-transfected cells $(n=6)$. Within 5 weeks, solid tumors were visible in right flank of all 6 mice (injected with scrambled shRNA-transfected cells), but not observed in any mouse injected with PSMA7 shRNA-transfected cells (Fig. 3C). These results showed that PSMA7 depletion has a strong tumor-suppressive ability in vitro and in vivo. 
(A) RT-PCR

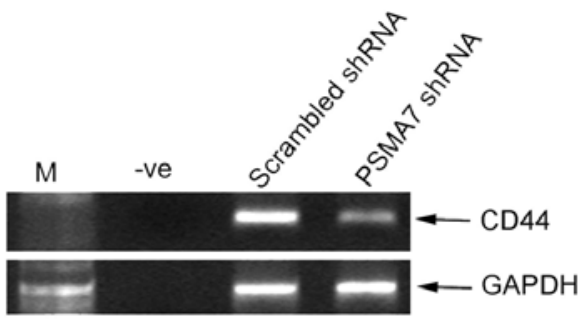

(B) Western blot

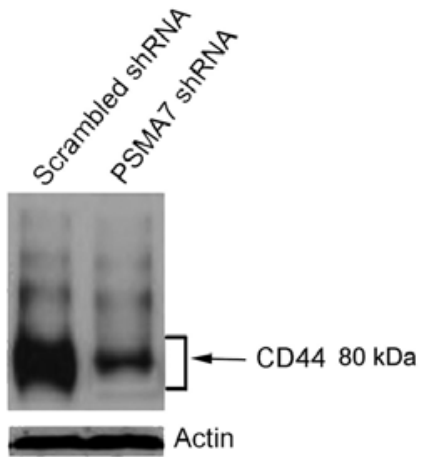

Figure 4. PSMA7 depletion significantly inhibited CD44 expression in RKO cells. CD44 mRNA (A) and protein (B) expression were decreased in PSMA7 shRNA-transfected RKO cells compared with scrambled shRNA-transfected RKO cells.

PSMA7 knockdown inhibited CD44 expression. To address the possible molecular basis contributing to the effects of PSMA7 depletion, we examined the effect of PSMA7 depletion on the expression of a panel of MMPs (MMP1, MMP2, MMP3, MMP7, MMP8, MMP9, MMP11, TIMP1, TIMP2, TIMP3, TIMP4) and an important cell adhesion molecule CD44. We firstly checked their expression by semi-quantitative RT-PCR, among them only TIMP1 and CD44 expressed in RKO cells, the others were not expressed in PSMA7 shRNAtransfected cells or in scrambled shRNA-transfected cells (data not shown). However, only CD44 mRNA expression was decreased clearly in PSMA7 shRNA-transfected cells compared with scrambled shRNA-transfected cells (Fig. 4A), while TIPM1 mRNA expressed equally in PSMA7 shRNAtransfected cells and scrambled shRNA-transfected cells (data not shown). CD44 expression was also decreased at the protein level in PSMA7 shRNA-transfected cells (Fig. 4B).

\section{Discussion}

We recently reported the relationship between PSMA7 expression and the clinicopathological features of colorectal cancer and suggested that PSMA7 may play an important role in the colorectal cancer progression and evaluation of PSMA7 expression in primary colorectal cancer at the time of surgery might be a valuable tool in defining patients with a high risk of developing liver metastasis. However, it is unknown how PSMA7 critically regulates the metastatic behavior of colorectal cancer cells.

In this study, we employed the RNA interference technology to knock down the PSMA7 gene in human colon cancer cell line RKO and analyzed its effect and explored the involved mechanisms. We found that knockdown of PSMA7 by shRNA in RKO cells inhibited their anchorage-independent growth and cell migration and invasion. Moreover, PSMA7 depletion was able to strongly suppress the in vivo tumorigenic ability of RKO cells.

Previously, there have been several studies on cellular regulatory proteins and viral proteins that interact with PSMA7 and participate in the proteasome-dependent regulation $(3,4)$. The hypoxia-inducible factor- $1 \alpha$ (HIF-1 $\alpha$ ) was identified as the first cellular target of PSMA7 and is an important transcription factor in the cellular response to oxygen tension (5). However in our study, after detecting HIF-1 $\alpha$ protein expression by Western blot analysis, no HIF-1 $\alpha$ was expressed in stable PSMA7 shRNA and scrambled shRNA-transfected RKO cells (data not shown).

Tumor cell invasion involves the movement of cells across the extracellular matrix (ECM). CD44 is a transmembrane cell-surface adhesion molecule that is synthesized in multiple isoforms because of alternative splicing of its pre-mRNA. CD44 has been found to support anchorage-independent growth in vitro and tumor growth and metastasis in experimental models of solid cancers (6-8), where it inhibited tumor growth in yet other models $(9,10)$. The precise role played by CD44 in tumorigenesis has thus remained unclear. On the other hand, the movement of cells into a tightly woven extracellular matrix may also require an active proteolytic system, which can cleave a path for cell migration. Among the ECM-degrading proteases, a great deal of attention has been paid to the MMP family. So we examined the effect of PSMA7 depletion on the expression of a panel of MMPs and the important cell adhesion molecule CD44. We found PSMA7 depletion may inhibit CD44 expression directly or indirectly.

Earlier studies employing the various CD44 models suggested that CD44 plays important roles in colon cancer, a tumor consistently characterized by overexpression of CD44. CD44 offers most resistance to apoptosis in both mouse and human colon (11-13). CD44 may also be involved in the directional motility of human colon cancer cells (14) and mechanistically regulate cofilin thereby altering the processes of cell migration (15). Moreover, recent studies have shown that it is a characteristic phenotype of tumor stem cells (16-18). However, the regulation of mechanism of CD44 protein expression remain unclear. In this study we found that PSMA7 expression positively correlated with CD44 expression. PSMA7 (also known as XAPC7, RC6-1, and HSPC in mammals) is one of the 7 proteasome $\alpha$-subunits. Accumulating evidence indicates that the proteasome not only plays a proteolytic role in protein degradation but also plays a non-proteolytic role in transcription elongation, nuclear excision repair and protein trafficking (19-23). Recently Godar et al (24) reported that growth-inhibitory and tumorsuppressive functions of p53 depend on its repression of CD44 expression, while Bae et al (25) found that regulation of early growth response 1 (Egr-1) gene was associated with the proteasome component $\mathrm{C} 8$. They are the important transcript factors of CD44 gene and also the short-lived proteins $(26,27)$. Since the selective degradation of many short-lived proteins in eukaryotic cells is carried out by the ubiquitin-dependent 26S proteasome (28). We hypothesized that PSMA7 may regulate CD44 expression directly or indirectly.

In conclusion, our results indicate that CD44 may be one of the down-stream elements in PSMA7-associated inhibition 
of colorectal cancer cell tumorigenicity and metastasis. Further investigation is necessary to elucidate the detail mechanisms and/or correlations between PSMA7 and CD44. Genetic or pharmacological inhibition of PSMA7 may therefore be a beneficial strategy in the treatment of colorectal cancer patients.

\section{Acknowledgements}

This study was supported by the Natural Science Foundation of Zhejiang Province, No. Y206090.

\section{References}

1. Grady WM and Carethers JM: Genomic and epigenetic instability in colorectal cancer pathogenesis. Gastroenterology 135: 1079-1099, 2008.

2. Hu XT, Chen W, Wang D, et al: The proteasome subunit PSMA7 located on the 20q13 amplicon is overexpressed and associated with liver metastasis in colorectal cancer. Oncol Rep 19: 441-446, 2008.

3. Zhang Z, Torii N, Furusaka A, Malayaman N, Hu Z and Liang TJ: Structural and functional characterization of interaction between hepatitis B virus X protein and the proteasome complex. J Biol Chem 275: 15157-15165, 2000.

4. Su K, Yang X, Roos MD, Paterson AJ and Kudlow JE: Human Sug $1 / \mathrm{p} 45$ is involved in the proteasome-dependent degradation of Sp1. Biochem J 348: 281-289, 2000.

5. Cho S, Choi YJ, Kim JM, et al: Binding and regulation of HIF-1alpha by a subunit of the proteasome complex, PSMA7. FEBS Lett 498: 62-66, 2001.

6. Barbour AP, Reeder JA, Walsh MD, et al: Expression of the CD44v2-10 isoform confers a metastatic phenotype: importance of the heparan sulfate attachment site CD44v3. Cancer Res 63: 887-892, 2003.

7. Weber GF, Bronson RT, Ilagan J, et al: Absence of the CD44 gene prevents sarcoma metastasis. Cancer Res 62: 2281-2286, 2002 .

8. Yu Q, Toole BP and Stamenkovic I: Induction of apoptosis of metastatic mammary carcinoma cells in vivo by disruption of tumor cell surface CD44 function. J Exp Med 186: 1985-1996, 1997.

9. Gao AC, Lou W, Dong JT and Isaacs JT: CD44 is a metastasis suppressor gene for prostatic cancer located on human chromosome 11p13. Cancer Res 57: 846-849, 1997.

10. Schmits R, Filmus J, Gerwin N, et al: CD44 regulates hematopoietic progenitor distribution, granuloma formation, and tumorigenicity. Blood 90: 2217-2233, 1997.

11. Lakshman M, Subramaniam V, Rubenthiran U and Jothy S: CD44 promotes resistance to apoptosis in human colon cancer cells. Exp Mol Pathol 77: 18-25, 2004.
12. Lakshman M, Subramaniam V and Jothy S: CD44 negatively regulates apoptosis in murine colonic epithelium via the mitochondrial pathway. Exp Mol Pathol 76: 196-204, 2004.

13. Lakshman M, Subramaniam V, Wong S and Jothy S: CD44 promotes resistance to apoptosis in murine colonic epithelium. $\mathrm{J}$ Cell Physiol 203: 583-588, 2005.

14. Wong K, Rubenthiran U and Jothy S: Motility of colon cancer cells: modulation by CD44 isoform expression. Exp Mol Pathol 75: 124-130, 2003.

15. Subramaniam V, Vincent IR and Jothy S: Upregulation and dephosphorylation of cofilin: modulation by CD44 variant isoform in human colon cancer cells. Exp Mol Pathol 79: 187-193, 2005.

16. Jin L, Hope KJ, Zhai Q, Smadja-Joffe F and Dick JE: Targeting of CD44 eradicates human acute myeloid leukemic stem cells. Nat Med 12: 1167-1174, 2006.

17. Krause DS, Lazarides K, von Andrian UH and Van Etten RA: Requirement for CD44 in homing and engraftment of BCRABL-expressing leukemic stem cells. Nat Med 12: 1175-1180, 2006.

18. Shipitsin M, Campbell LL, Argani P, et al: Molecular definition of breast tumor heterogeneity. Cancer Cell 11: 259-273, 2007.

19. Pickart CM and VanDemark AP: Opening doors into the proteasome. Nat Struct Biol 7: 999-1001, 2000.

20. Shenoy SK, McDonald PH, Kohout TA and Lefkowitz RJ: Regulation of receptor fate by ubiquitination of activated beta 2adrenergic receptor and beta-arrestin. Science 294: 1307-1313, 2001.

21. Yu A and Malek TR: The proteasome regulates receptor-mediated endocytosis of interleukin-2. J Biol Chem 276: 381-385, 2001.

22. Ferdous A, Gonzalez F, Sun L, Kodadek T and Johnston SA: The $19 \mathrm{~S}$ regulatory particle of the proteasome is required for efficient transcription elongation by RNA polymerase II. Mol Cell 7: 981-991, 2001.

23. Gillette TG, Huang W, Russell SJ, Reed SH, Johnston SA and Friedberg EC: The 19S complex of the proteasome regulates nucleotide excision repair in yeast. Genes Dev 15: 1528-1539, 2001.

24. Godar S, Ince TA, Bell GW, et al: Growth-inhibitory and tumorsuppressive functions of 553 depend on its repression of CD44 expression. Cell 134: 62-73, 2008.

25. Bae MH, Jeong CH, Kim SH, et al: Regulation of Egr-1 by association with the proteasome component $\mathrm{C} 8$. Biochim Biophys Acta 1592: 163-167, 2002.

26. Recio JA and Merlino G: Hepatocyte growth factor/scatter factor induces feedback up-regulation of CD44v6 in melanoma cells through Egr-1. Cancer Res 63: 1576-1582, 2003.

27. Zavrides HN, Zizi-Sermpetzoglou A, Panousopoulos D, Athanasas G, Elemenoglou I and Peros G: Prognostic evaluation of CD44 expression in correlation with bcl-2 and p53 in colorectal cancer. Folia Histochem Cytobiol 43: 31-36, 2005.

28. Hershko A and Ciechanover A: The ubiquitin system. Annu Rev Biochem 67: 425-479, 1998. 